\title{
Cirugía en tuberculosis pulmonar multirresistente
}

\author{
Ángela Tobón ${ }^{1,2}$, Lizeth Paniagua ${ }^{2,3}$, Marcela Henao ${ }^{2,3}$, Vivian Arcila ${ }^{1,4}$, José Maya ${ }^{1}$, \\ Fernando Bedoya ${ }^{1}$, Bernardo Muñoz ${ }^{1}$ \\ ${ }^{1}$ Hospital La María, Medellín, Colombia. \\ ${ }^{2}$ Corporación para Investigaciones Biológicas, Medellín, Colombia. \\ ${ }^{3}$ Facultad de Medicina, Universidad Pontificia Bolivariana, Medellín, Colombia. \\ ${ }^{4}$ Hospital Universitario San Vicente de Paúl, Medellín, Colombia.
}

La aparición de Mycobacterium tuberculosis multirresistente constituye un serio problema para el control de la tuberculosis, por lo que se ha considerado que la resección quirúrgica del tejido pulmonar afectado, junto con el tratamiento médico adecuado, podría proporcionar la curación en algunos pacientes con tuberculosis pulmonar multiresistente. En este estudio de tipo descriptivo, retrospectivo, se evaluaron los resultados clínicos y bacteriológicos de la resección quirúrgica en un grupo seleccionado de pacientes con tuberculosis pulmonar multirresistente. Se revisaron las historias clínicas del Hospital La María de Medellín de 1990 a 2000, y se encontró que se habían sometido a cirugía 73 pacientes con diagnóstico de tuberculosis durante este periodo, 28 de los cuales tenían como indicación quirúrgica tuberculosis multirresistente (resistencia a isoniacida y rifampicina), 21 de los cuales habían recibido tratamiento prequirúrgico. En 14 (50\%) se había practicado lobectomía superior y en $10(36,7 \%)$, neumonectomía. Todos recibieron tratamiento posquirúrgico por un periodo promedio de 12,5 meses. En 88,9\% (25/27) de los casos, la baciloscopia fue negativa después de 6 semanas de la cirugía y hasta finalizar el tratamiento antituberculoso; 6 pacientes presentaron nuevamente baciloscopia positiva, aunque 4 tuvieron baciloscopia negativa después de un nuevo esquema de tratamiento. Los pacientes, de acuerdo con las normas del ministerio, fueron seguidos con baciloscopia mensual y no con cultivo como es lo indicado en los casos de multirresistencia. La cirugía junto con el tratamiento médico adecuado constituye una buena alternativa de curación para algunos pacientes con tuberculosis pulmonar multirresistente.

Palabras clave: cirugía, tuberculosis pulmonar, multirresistencia.

\section{Surgical treatment of multiresistant lung tuberculosis}

Drug resistance has become a major problem in the treatment of tuberculosis. Pulmonary resection in combination with chemotherapy appears to be an effective measure for the treatment of multi-drug resistant pulmonary tuberculosis.

A retrospective review was performed of the medical and laboratory findings of 28 patients with multi-drug resistant tuberculosis who underwent pulmonary resection for pulmonary tuberculosis between January 1990 and December 2000 at La Maria Hospital, Medellín. Twenty-one of them had medical therapy before surgery; 14 patients underwent upper lobectomy and 10 patients pneumonectomy). The AFB negative sputum conversion rate was $88.9 \%(25 / 27)$ after surgery, during an average of 6 weeks. Bacteriological relapses were confirmed in 6 of 27, 4 of these 6 had AFB negative sputum. Twenty-eight patients had medical therapy after surgery. For selected patients, pulmonary resection in combination with chemotherapy should be considered an effective measure for treatment of multi-drug resistant pulmonary tuberculosis.

Key words: surgery, multi-drug resistant, pulmonary tuberculosis. 
La Organización Mundial de la Salud (OMS) estima que existen en el mundo 50 millones de personas que están infectadas por Mycobacterium tuberculosis multirresistente (1).

En Colombia, el primer informe de resistencia global fue publicado en 1981 cuando se demostró que, para $1980,63,1 \%$ de los casos en los que se sospechaba resistencia debida a falla terapéutica, lo eran por lo menos a un medicamento (2). En el estudio coordinado por la OMS y la IUALTD (1994-1997), realizado a escala mundial, Colombia no aportó información sobre tuberculosis multirresistente en los pacientes con historia de tratamiento previo en el primer estudio (3).

En el departamento de Antioquia, entre 1995 y 1997, en una población de 172 pacientes que habían recibido tratamiento antituberculoso por más de un mes, se reportó resistencia a un medicamento, por lo menos, en $65,7 \%$ de los casos: $9 \%$ fueron resistentes a un medicamento, $16 \%$ a dos, $20 \%$ a tres y $17 \%$ a cuatro (4). La multirresistencia y la resistencia combinada (rifampicina-isoniacida) se presentó en $74,6 \%$ de los pacientes.

A pesar de que el tratamiento acortado supervisado permite la curación hasta del 95\% de los casos susceptibles, la aparición de multirresistencia atenta contra el éxito de esta estrategia y aun contra el control global de la tuberculosis (5). Desde el punto de vista de salud pública, un tratamiento incompleto, irregular y pobremente supervisado es peor que no hacer ningún tratamiento $(6,7)$.

La aparición de cepas multirresistentes, definida como M. tuberculosis resistente a dos de los agentes más importantes en el control de la enfermedad tuberculosa - isoniacida y rifampicina (8) - representa un serio problema para el control de la tuberculosis, el que podría, además, transformarse en una pandemia incontrolable (6).

Correspondencia:

Lizeth A. Paniagua, Carrera 72 A No.78-B-141, Medellín. Colombia

Teléfono: (572) 441 0855, extensión 229; fax: (572) 4415514 Ipaniagua@cib.org.co

Recibido: 23/07/03; aceptado: 12/02/04
Las causas más frecuentes de esta resistencia son los tratamientos inadecuados, el deterioro en los programas de control de la enfermedad y la transmisión de cepas resistentes a poblaciones susceptibles $(9,10)$.

Cuando se conoce o se sospecha multirresistencia, el tratamiento inicial debe incluir, al menos, 4 medicamentos para los que se haya demostrado sensibilidad y, preferiblemente, que el paciente no haya recibido anteriormente (11). La duración de esta terapia depende de los agentes utilizados y de la extensión de la enfermedad, pero, generalmente, debe hacerse por 18 a 24 meses luego de hacerse negativos los cultivos (12). La resección del tejido pulmonar afectado parece ser una buena alternativa como terapia adjunta en el tratamiento de la tuberculosis multirresistente en aquellos pacientes con lesiones pulmonares localizadas y dificultad en la consecución de los medicamentos de segunda línea. Ésta permitiría la disminución de la carga bacilar, principalmente, en aquellos pacientes con presencia de lesiones cavitarias, destrucción pulmonar con fibrosis y resistencia a los medicamentos de primera línea $(13,14)$.

La intervención quirúrgica en el tratamiento de la tuberculosis fue utilizada en gran escala en el siglo XIX, frecuentemente con colapso de la región del pulmón que contenía la lesión cavitaria. Sin embargo, con el advenimiento de la quimioterapia en 1940, esta práctica fue abandonada dada la alta proporción de curación obtenida con el tratamiento farmacológico.

Cuatro décadas después de iniciada la terapia antibiótica, emergieron cepas de M. tuberculosis resistentes a los medicamentos de primera línea (15). Es así como nuevamente surge la cirugía junto con el tratamiento médico como una buena herramienta para brindarles curación a estos pacientes (12). Además, se han encontrado otras indicaciones para la cirugía en pacientes con tuberculosis, como la aparición de secuelas de fibrotórax, hemoptisis, fístulas broncopleurales, abscesos y lesiones cavitarias persistentes a pesar del tratamiento médico adecuado $(16,17)$.

No existen publicaciones en Colombia sobre la experiencia de la cirugía como terapia 
coadyuvante en el tratamiento de la tuberculosis multirresistente. En este estudio se evaluaron los resultados clínicos y bacteriológicos de la cirugía como terapia coadyuvante en el tratamiento de la tuberculosis multirresistente en un grupo seleccionado de pacientes del Hospital La María de Medellín (Antioquia), el cual es un centro de referencia para la atención de los pacientes con tuberculosis de difícil manejo.

\section{Pacientes y métodos}

Se realizó un estudio descriptivo, retrospectivo. La fuente primaria de la información fueron las historias clínicas de los archivos del Hospital La María, de pacientes con tuberculosis pulmonar intervenidos quirúrgicamente entre enero de 1990 y diciembre de 2000. Además, se revisaron las historias clínicas de los pacientes que no fueron intervenidos quirúrgicamente por presentar compromiso bilateral serio y difuso.

Los pacientes sometidos a cirugía presentaban lesiones pulmonares bien localizadas y su función cardiopulmonar no estaba gravemente comprometida.

El manejo médico-quirúrgico de los pacientes se llevó a cabo en el Hospital La María, antiguo sanatorio de tuberculosis y, actualmente, hospital general con atención neumológica de tercer nivel, y centro de referencia para el manejo de los pacientes con tuberculosis complicada.

En todos los pacientes la cirugía fue practicada por el mismo grupo quirúrgico del Hospital La María y por el mismo cirujano de tórax con alta experiencia en cirugía de pacientes con tuberculosis pulmonar.

Las pruebas de sensibilidad se hicieron en el Laboratorio Departamental de Salud de Antioquia por el método de Canneti. Los medicamentos evaluados fueron la estreptomicina, la rifampicina, la isoniacida, el etambutol, la etionamida y la tioacetazona durante el tiempo del estudio.

Se construyó una base de datos en la que se tuvieron en cuenta las características sociodemográficas y ciertas variables relacionadas con el tratamiento médico y quirúrgico llevado a cabo en dichos pacientes.
Se analizó la evolución clínica y bacteriológica de los pacientes consignada en las historias clínicas y, en forma complementaria, algunos pacientes se citaron con el fin de ampliar los datos. La información se recopiló con el programa Microsoft Excel 2000 y se realizó un análisis estadístico descriptivo.

\section{Resultados}

Entre enero de 1990 y diciembre de 2000, 73 pacientes con diagnóstico de tuberculosis pulmonarse fueron sometidos a cirugía de tórax. Cuarenta y cinco $(61,6 \%)$ fueron intervenidos quirúrgicamente por presentar complicaciones de una tuberculosis antigua o en tratamiento, de los cuales, $15(33,3 \%)$ presentaban hemoptisis recurrente, $16(35,6 \%)$ fibrotórax con persistencia de actividad tuberculosa, 6 (13,3\%) presentaban lesión cavitaria persistente sin respuesta al tratamiento médico, $3(6,66 \%)$ tenían fístula broncopleural persistente y $5(11,1 \%)$ fueron intervenidos por masa pulmonar de etiología desconocida y, después de la cirugía, se estableció el diagnóstico de tuberculosis. Los otros $28(38,4 \%)$ pacientes tenían como indicación quirúrgica la falta de respuesta al tratamiento por multirresistencia a medicamentos antituberculosos.

De estos 28 pacientes, 21 eran hombres y 7 mujeres. La edad promedio fue de 37,3 años, con un rango de edad entre 18 y 73 años. Se encontró que $5(17,8 \%)$ tenían diabetes mellitus como enfermedad comórbida. De acuerdo con los hallazgos radiológicos, $13(46,4 \%)$ pacientes presentaban lesiones bilaterales.

Antes de la intervención quirúrgica, 19 (67,8\%) pacientes recibieron un nuevo esquema de tratamiento antituberculoso por un tiempo promedio de 1,5 meses y $2(7,2 \%)$ continuaron con el esquema que venían recibiendo, mientras que en $7(25 \%)$ el esquema de tratamiento antituberculoso no estaba especificado en la historia clínica. En 23 (82,2\%) pacientes, los medicamentos suministrados antes de la cirugía fueron rifampicina e isoniacida; en 19 (67,8\%) pacientes, pirazinamida y etambutol. Otros medicamentos, como estreptomicina, etionamida, tioacetazona y ofloxacina, se emplearon en un menor número de pacientes. 
Con respecto al tipo de cirugía, se realizó lobectomía superior en 14 (50\%) pacientes y neumonectomía izquierda en $6(21,4 \%)$; se practicó neumonectomía derecha en 4 (14,3\%), segmentectomía en $3(10,7 \%)$ y toracoplastia en $1(3,6 \%)$.

En todos los pacientes, las historias clínicas informaron el aislamiento en cultivo de $M$. tuberculosis con existencia de multirresistencia en las pruebas de sensibilidad. Se reportó resistencia a dos medicamentos en $28,6 \%$, a 3 medicamentos en $32,2 \%$ de los pacientes, a cuatro medicamentos el $25 \%$ y a 5 el $14,2 \%$. En el cuadro 1 se describe la frecuencia de resistencia aislada a cada uno de los medicamentos.

Luego de la cirugía, todos los pacientes, recibieron tratamiento farmacológico por un tiempo promedio de 12,5 meses, con un rango entre 6 y 28 meses. Los medicamentos recibidos están descritos en el cuadro 2.

Se indica en este grupo de pacientes realizar el seguimiento con cultivos de esputo ya que la baciloscopia positiva no garantiza la viabilidad de la micobacteria.

Sólo en 7 pacientes se pudo hacer el seguimiento bacteriológico con cultivo de esputo debido a que la mayoría de los pacientes continuaron su manejo en otras instituciones clínicas de la ciudad. En este grupo, la negativización del cultivo se logró en un promedio de 12,4 semanas, con un rango de 4 a 20 semanas.

En los demás pacientes, el seguimiento con baciloscopia mostró negativización en un

Cuadro 1. Frecuencia de resistencia por medicamento en 28 pacientes operados con tuberculosis pulmonar resistente.

\begin{tabular}{llr} 
Medicamento & \multicolumn{2}{c}{ *Pacientes } \\
\hline Isoniacida & \% \\
Rifampicina & 28 & 100 \\
Estreptomicina & 28 & 100 \\
Etambutol & 21 & 75 \\
Pirazinamida & 9 & 32,1 \\
Etionamida & 2 & 7,2 \\
\hline
\end{tabular}

*Un paciente podía ser resistente a varios medicamentos simultáneamente.
Cuadro 2. Frecuencia del uso de medicamentos antituberculosos posterior a la cirugía.

\begin{tabular}{lcc}
\hline Medicamento & \multicolumn{2}{c}{ Pacientes } \\
\hline Rifampicina & $\mathbf{n}$ & $\%$ \\
Isoniacida & 28 & 100 \\
Pirazinamida & 28 & 100 \\
Etambutol & 24 & 85,7 \\
Etionamida & 17 & 60,7 \\
Estreptomicina & 10 & 35,7 \\
Tioacetazona & 6 & 21,4 \\
Ciprofloxacina & 6 & 21,4 \\
Amikacina & 4 & 14,3 \\
Sin dato & 1 & 3,6 \\
\hline
\end{tabular}

promedio de 6 semanas, con un rango entre 1 y 24 semanas.

De los 28 pacientes, uno murió 48 horas después de la cirugía por falla respiratoria aguda y 27 $(96,4 \%)$ sobrevivieron. De éstos, $3(11,1 \%)$ nunca presentaron baciloscopias negativas y $24(88,9 \%)$ sí lo hicieron. Sin embargo, de los anteriores, 6 $(25 \%)$ presentaron nuevamente baciloscopia positiva durante el tratamiento antituberculoso en un tiempo posquirúrgico promedio de 19,3 meses, con rango entre 11 y 36 meses.

De 31 pacientes con multirresistencia que no fueron intervenidos quirúrgicamente por presentar compromiso bilateral serio y difuso y que sólo recibieron tratamiento farmacológico, $12(38,7 \%)$ no curaron, $7(23 \%)$ sí lo hicieron con nuevos esquemas terapéuticos, 6 (20\%) abandonaron la terapia y $6(20 \%)$ no tenían datos consignados en la historia clínica.

\section{Discusión}

Uno de los mayores problemas para el control de la tuberculosis es la aparición y el aumento de micobacterias resistentes a la terapia, hecho que la convierte en un serio problema de salud pública, no sólo por el aumento en el número de casos de difícil y costoso tratamiento, sino por la gran mortalidad que este fenómeno produce (18). En efecto, en una cuarta parte de los enfermos, la mayoría en etapas productivas de la vida, la terapia inadecuada conduce a la muerte hasta en el $50 \%$ de los casos $(17,18)$.

El aumento de la resistencia se debe a varias causas, entre ellas, la falta de adherencia al 
tratamiento por parte de los pacientes, el deterioro de los programas de control de la enfermedad y los tratamientos inadecuados $(6,7,18)$.

El problema se hace mayor cuando esta resistencia se presenta a rifampicina e isoniacida (multirresistencia), los dos medicamentos de mayor efectividad en el esquema de tratamiento actual, ya que obliga a la utilización de medicamentos de segunda línea, los cuales son menos eficaces, más costosos y con efectos adversos más frecuentes.

En la mayoría de los países en desarrollo, incluida Colombia, la consecución de medicamentos de segunda línea es difícil, el suministro de algunos de ellos - como las quinolonas - por parte del servicio de salud es complicado por no formar parte de la lista de medicamentos del plan obligatorio de salud (POS) y su compra por parte de los pacientes es prácticamente imposible dadas sus condiciones económicas. Los pacientes continúan recibiendo tratamientos inefectivos por varios años y excretando bacilos multirresistentes con las implicaciones epidemiológicas graves que esto representa. Esta situación ha obligado a buscar alternativas terapéuticas que permitan controlar este problema infeccioso.

Actualmente, algunos proponen para el tratamiento de la tuberculosis multirresistente la cirugía de resección pulmonar asociada con un tratamiento farmacológico adecuado, como una alternativa importante para el control de esta patología (19-21). Esto parece comprobarse en el presente estudio, en el cual de 28 pacientes multirresistentes a los medicamentos antituberculosos, 22 (78,5\%) continuaron con baciloscopia negativa durante el tiempo de seguimiento posterior a la cirugía. De estos 28 pacientes, 21 eran hombres y la mayoría estaban en etapas productivas de la vida (37,3 años). Dicho hallazgo resalta la importancia de identificar tempranamente los pacientes que podrían beneficiarse de la cirugía para limitar las repercusones económicas negativas que produce esta enfermedad $(18,22)$.

No fue posible establecer correlación alguna entre la baciloscopia y los cultivos, ya que estos últimos no se contemplan en el control mensual de los pacientes en tratamiento, lo cual impide evaluar completamente el resultado de curación en los pacientes con multirresistencia sometidos a cirugía.

En nuestro estudio, los pacientes recibieron tratamiento prequirúrgico por un tiempo promedio de 1,5 meses, tiempo similar al recomendado en otros estudios (2 meses) (23). La cirugía se debe llevar a cabo tempranamente durante la terapia farmacológica con el fin de evitar mayor deterioro y preservar la mayor cantidad posible de parénquima pulmonar $(24,25)$.

De los 28 pacientes sometidos a cirugía, 13 $(46,4 \%)$ presentaban lesiones bilaterales; sin embargo, en la mayoría de estos pacientes las zonas más afectadas estaban suficientemente localizadas en uno de los pulmones lo cual permitió su resección. Ésta se practicó con el fin de disminuir la carga bacteriana por medio de la resección de cavernas, conocidas con alto número de micobacterias alojadas dentro de ellas $\left(10^{7}-10^{9}\right)$ y pobre penetración de los antibióticos en esta zona rodeada por gran fibrosis $(24,25)$.

Con respecto a la evolución bacteriológica en este grupo de pacientes, se encontró que 3 pacientes persistieron con baciloscopias positivas, 25 $(88,9 \%)$ pacientes prsentaron resultados negativos después de la cirugía en un tiempo promedio de 6 semanas y persistieron así hasta el final del tratamiento. De éstos, 6 presentaron nuevamente baciloscopia positiva durante el tratamiento en un tiempo promedio de 19,3 meses, sin poder definirse claramente si representaban recaída de la enfermedad o reinfección. De estos 6 pacientes, 4 presentaron nuevamente baciloscopia negativa con un nuevo esquema de tratamiento (figura 1). Estos datos son semejantes a los resultados informados en otros estudios $(26,27)$. Infortunadamente, el presente estudio por la limitación en la realización de los cultivos, no permite determinar el índice de curación en el grupo de pacientes, ya que se requiere de este examen para determinarla. Sólo fue posible realizarlo en un número menor de pacientes.

Al analizar este grupo de 6 pacientes, se puede resaltar que 3 de ellos eran diabéticos, posiblemente presentaron recaída por pobre 


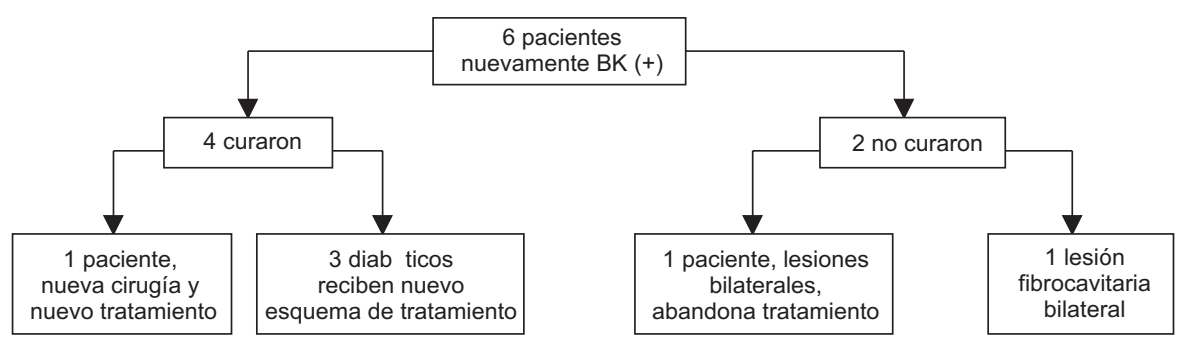

Figura 1. Evolución clínica de 6 pacientes nuevamente con baciloscopia positiva.

respuesta al tratamiento antituberculoso al tener alteraciones de tipo inmune y mayor riesgo para multirresistencia (28-30). Dos pacientes presentaban lesiones fibrocavitarias bilaterales y uno abandonó el tratamiento luego de la cirugía.

Con respecto al tratamiento antituberculoso posterior a la cirugía, se ha descrito la necesidad de suministrarlo entre 12 y 18 meses $(11,20,25)$; en nuestro estudio, la duración promedio fue de 12,5 meses.

Sólo un paciente $(3,6 \%)$ presentó fístula broncopleural como complicación posquirúrgica y un paciente murió 48 horas dspués de la cirugía, lo que muestra la baja morbimortalidad en este tipo de intervención $(24,26)$.

Se llama la atención al tipo de medicamentos suministrados luego de la cirugía, pues sólo un porcentaje bajo de los pacientes recibieron medicamentos de segunda línea, debido a su difícil consecución en Colombia. La mayor parte de ellos fueron tratados nuevamente con los cuatro medicamentos de primera línea, excepto la estreptomicina por razón de su límite de dosis acumulada. Éstos se acompañaron de etambutol y etionamida en un número importante de pacientes $(60,7 \%$ y $35,7 \%$, respectivamente) y de quinolonas y amikacina en un número menor.

Esto refuerza aún más la importancia de la disminución de la carga bacilar mediante la resección quirúrgica del tejido pulmonar afectado, para lograr su posterior esterilización con la terapia farmacológica (27).

En este trabajo pudimos observar cómo en este grupo de pacientes, la cirugía como terapia adjunta a los medicamentos antituberculosos mostró ser una buena alternativa en el tratamiento de tuberculosis multirresistente, ya que la morbimortalidad fue mínima y un alto número de pacientes, 22 de 28 (78,5\%), presentaron baciloscopias negativas por tiempo prolongado, con mejoría de su calidad de vida y disminución de la cadena de transmisión de la enfermedad.

Se han informado en otros estudios resultados que demuestran el papel importante de la cirugía en este grupo de pacientes $(21,24,25,31,32)$. Kiar y colaboradores reportaron que los 27 pacientes incluidos en su estudio, se negativizaron luego de la cirugía en un tiempo promedio de 4 meses. Iseman y colaboradores reportaron que 25 (92,6\%) de 27 pacientes habían tenido control bacteriológico de la enfermedad después de la cirugía y Sook-Whan y colaboradores reportaron que de 27 pacientes, 22 (81,5\%) presentaron conversión de esputo inmediatamente posterior a la cirugía y otros $4(14,8 \%)$ lo hicieron posterior al retratamiento. Todos ellos con cultivos negativos como forma de control luego de la terapia combinada.

Un aspecto importante para tener en cuenta es el alto costo que representa el tratamiento farmacológico prolongado con múltiples medicamentos y el costo adicional de la cirugía, cerca de US \$700, cuando el paciente no tiene subsidio para el pago de su tratamiento. Por lo cual insistimos en la necesidad de prevenir la aparición de multirresistencia como la medida más importante para el control de la enfermedad tuberculosa.

En el futuro podrían realizarse estudios similares con seguimientos de control a través de cultivos, para establecer en forma precisa el valor de la 
cirugía en la terapia de los pacientes con tuberculosis multirresistente.

\section{Agradecimientos}

Este estudio se pudo realizar gracias al apoyo del personal del Hospital La María de Medellín, especialmente, al personal del archivo.

\section{Referencias}

1. Caminero L. Origen, presente y futuro de las resistencias en tuberculosis. Arch Bronconeumol 2001; 37:35-42.

2. Orozco L, Aparicio G, Quintero O, Giraldo E, Ulloa I, León C. Resistencia de M. tuberculosis a las drogas. Biomédica 1981;1:130-4.

3. World Health Organization/IUATLD. Anti-tuberculosis drug resistance in the world. Report No. 2 Prevalence and trends. Global Project on Anti-tuberculosis Drug Resistance Surveillance. Geneva: WHO; 2000.

4. Estrada S, Gil M, Rodríguez M, Posada P, Álvarez H. Tuberculosis resistente en una población seleccionada del departamento de Antioquia (Colombia). Rev Panam Infectol 1998;2:91-6.

5. Kimerling $\mathbf{M}$, Kluge $\mathbf{H}$, Vezhnina $\mathbf{N}$, Lacovazzi $\mathbf{T}$, Demeulenaere $\mathrm{T}$, Portaels $\mathrm{F}$, et al. Inadequacy of the current WHO retreatment regimen in a central Siberian Prision. Int J Tuberc Lung Dis 1999;3:450-3.

6. Centers for Disease Control, Division of Tuberculosis Elimination. World TB Day, March 24, 2001. The history of world TB day. www.cdc.gov

7. Iseman M. Treatment of multi-drug resistant tuberculosis. N Engl J Med 1993;329:784-90.

8. Becerra MC, Bayona J, Freeman PE, Farmer PE, Kim JY. Redefining MDR-TB transmission 'hot spot'. Int J Tuberc Lung Dis 2000;4:387-94.

9. Mahmoundi A, Iseman M. Pitfalls in the care of patients with tuberculosis. JAMA 1993;270:65-8.

10. Goble M, Iseman M, Madsen L, Waite D, Ackerson L, Horsburg R. Treatment of 171 patients with pulmonary tuberculosis resistant to isoniazid and rifampin. N Engl J Med 1993;328:52.

11. WHO/CDC. Treatment of tuberculosis: guidelines for national programmes. Third edition. htpp: //who.int.gtb/ publications/ttgnp/index.html; 2003.

12. Crofton S. Directrices para el tratamiento de la tuberculosis farmacorresistente. Ginebra: OMS; 1997.

13. Bradford W, Daley C. Multiple drug resistant tuberculosis. Infect Dis Clin North Am 1998;12:157-72.

14. Treasure R, Seaworth B. Current role of surgery in Mycobacteriun tuberculosis. Ann Thorac Surg 1995;59: 1405-7.
15. Iseman M, Madsen L, Goble M, Pomerantz M. Surgical intervention in the treatment of pulmonary disease caused by drug-resistant Mycobacterium tuberculosis. Am Rev Respir Dis 1990;141:623-5.

16. Harrison L. Current aspects of the surgical management of tuberculosis. Surg Clin North Am 1980;8:883-95.

17. Freixinet J. Surgical indications for treatment of pulmonary tuberculosis. World J Surg 1997;21:475-9.

18. Floyd K, Blanc L, Raviglione M, Lee JW. Resources required for global tuberculosis control. Science 2002; 295:2040-1.

19. Drobniewski F, Eltringham I, Graham C, Magee J, Smith E, Watt B. A national study of clinical and laboratory factors affecting the survival of patients with multiple drug resistant tuberculosis in UK. Thorax 2002;57:815-6.

20. Pomerantz B, Cleveland J, Olson H, Pomerantz M. Pulmonary resection for multi-drug resistant tuberculosis. J Thorac Cardiovasc Surg 2001;121:44853.

21. Sung S, Kang C, Kim Y, Han S, Shim Y, Kim J. Surgery increased the chance of cure in multi-drug resistant pulmonary tuberculosis. Eur J Cardiothorac Surg 1999; 16:187-93.

22. Park SK, Lee CM, Heu HP, Song SD. A retrospective study for the outcome of pulmonary resection in 49 patients with multi-drug resistant tuberculosis. Int $\mathrm{J}$ Tuberc Lungs Dis 2002;6:143-9.

23. Kiar A. Role of surgery in multi-drug resistant tuberculosis: results of 27 cases. Eur $\mathrm{J}$ Cardiothorac Surg 1997;4:531-4.

24. Iseman M, Madsen L, Goble M, Pomerantz M. Surgical intervention in the treatment of pulmonary disease caused by drug-resistant Mycobacterium tuberculosis. Am Rev Respir Dis 1990;141:623-5.

25. Nakajima Y. Surgical treatment of multidrug resistant pulmonary tuberculosis cases. Kekkaku 1997;72:2534.

26. Pomerantz M. Surgical intervention in the treatment of pulmonary disease caused by drug-resistant Mycobacterium tuberculosis. Am Rev Respir Dis 1990; 141:623-5.

27. Chiang CY, Yu MC, Bai KJ, Suo J, Lin TP, Lee YC. Pulmonary resection in the treatment of patients with pulmonary multi-drug resistant tuberculosis in Taiwan. Int J Tuberc Lung Dis 2001;5:272-7.

28. Hansen L, Prakash U. Pulmonary complications in Diabetes Mellitus. Mayo Clin Proc 1989;64:791-9.

29. Mboussa J, Monabeka H, Kombo M, Yokolo D, YokaMbio A, Yala F. Course of tuberculosis in diabetics. Rev Pneumol Clin 2003;59:39-44. 
30. Bashar M, Alcabes P, Rom W, Condos R. Increased incidence of multidrug-resistant tuberculosis in diabetic patients on the Bellevue Chest Service,1987 to1997. Chest 2001;120:1514-9.

31. Golovchenko RN, Grigorian VA, Malygina VI. Lung resection in drug resistant tuberculosis patients. Probl Tuberk 2001;9:10-1.
32. Vancevicius V, Grazelis E, Serpytis J, Gineitis A. The surgical treatment of lung tuberculosis. Medicina (Kaunas) 2002;38(Suppl.2):30-2.

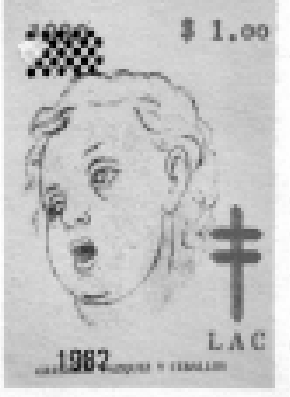

